\title{
TRENCH-ISOLATED BULK-TYPE PRESSURE SENSOR ON SILICON-ON-INSULATOR FOR HIGH-TEMPERATURE AND HIGH-PRESSURE DOWNHOLE APPLICATIONS
}

Elena Chan ${ }^{1}$, Dequan Lin $^{1,2,3}$, Lei Lu ${ }^{1,4}$, Kevin Chau ${ }^{1,2}$, and Man Wong ${ }^{1}$

${ }^{1}$ The Hong Kong University of Science and Technology, Hong Kong, China

${ }^{2}$ The Institute of Geology and Geophysics, Chinese Academy of Sciences, Beijing, China

${ }^{3}$ The University of Chinese Academy of Sciences, Beijing, China

${ }^{4}$ The HKUST Jockey Club Institute for Advanced Study, Hong Kong, China

\begin{abstract}
Presently reported is a dielectric-isolated bulk-type pressure sensor fabricated on silicon-on-insulator (SOI) that offers higher pressure capability than conventional diaphragm-type pressure sensors and wider temperature operating range than a recently reported junction-isolated bulk-type pressure sensor. The isolated piezoresistors are realized on the SOI device layer via trench etching and oxidation. Two types of trenches are formed: narrow ones that are completely filled with silicon dioxide and wide ones that are thermally oxidized on the sidewalls but partially filled. The pressure sensitivity is enhanced by relieving the transverse stress acting on selected piezoresistors isolated with the wide trenches.
\end{abstract}

\section{INTRODUCTION}

Resonant quartz pressure sensor (Fig. 1a) currently sets the benchmark for downhole pressure measurements. While MEMS pressure sensor with its relatively low cost and small size has the potential to replace the resonant quartz pressure sensor, it is still hindered by a number of limitations. A recent attempt [1] has been made to address some of the issues by replacing the conventional diaphragm-type with a bulk-type pressure sensor to handle the targeted high-pressure load ( $\geq 200 \mathrm{MPa}$ ), but the junction-isolation of the piezoresistors must be replaced by the presently demonstrated dielectric-isolation to handle the anticipated high operating temperature $\left(\geq 200^{\circ} \mathrm{C}\right)$.

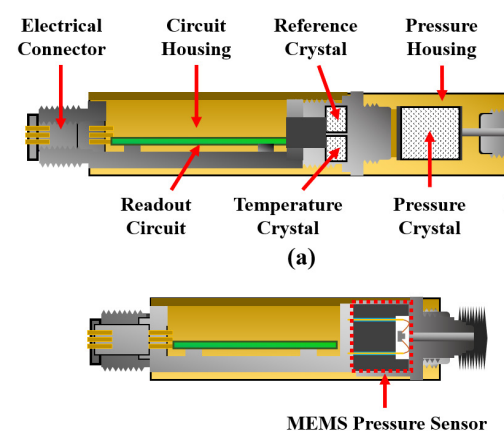

(b)

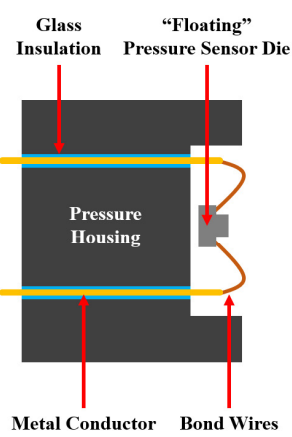

(c)
Figure 1: (a) Schematic diagram of a resonant quartz pressure sensor package. (b) Schematic diagram showing the quartz crystals replaced by a MEMS pressure sensor. (c) Magnified schematic of the MEMS pressure sensor die suspended by bond wires.

A higher pressure sensitivity is desirable for reducing the electrical offset and its associated temperature coefficient. To achieve this, selective trenching of the piezoresistors to release specific stress components is explored. In addition, the packageinduced stress that ultimately determines sensor repeatability, hence compensability, must also be minimized. Presently proposed is a MEMS pressure sensor designed as a direct drop-in replacement of the quartz resonator capsule in an inconel package that is proven for downhole applications (Fig. 1b). The MEMS sensor die is suspended only by its bond wires, hence largely relieved of the package-induced stress (Fig. 1c). This potentially allows accurate temperature compensation, despite the temperature coefficient of a piezoresistive silicon sensor being intrinsically two orders of magnitude higher than that of a resonant quartz sensor.

\section{PRINCIPLE OF OPERATION}

The bulk-type pressure sensor is formed by eutectically bonding a [110]-oriented SOI sensor wafer and a cap wafer under vacuum (Fig. 2). Two orthogonal pairs of piezoresistors respectively aligned to the [001] and [1 10$]$ orientations are realized in the center region on the sensor-side of the vacuum cavity and electrically configured to form a Wheatstone bridge.

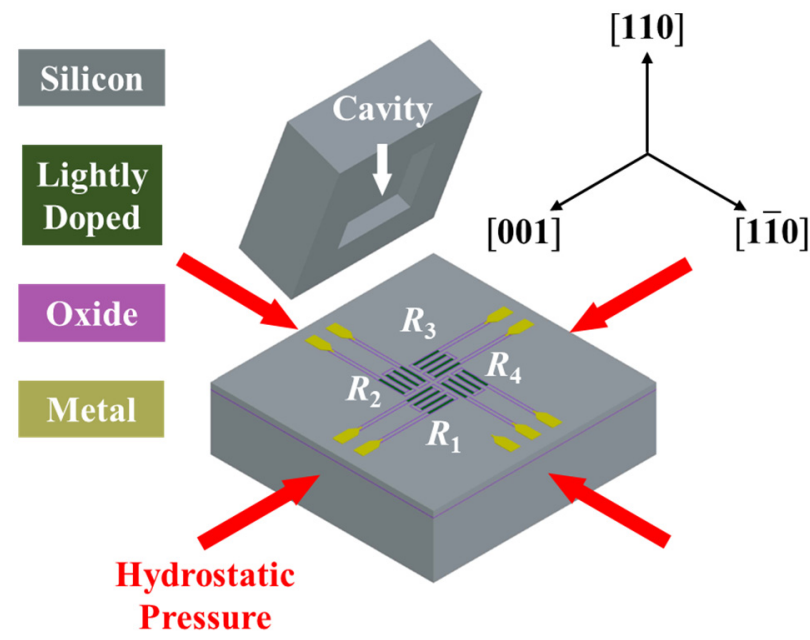

Figure 2: Bulk-type pressure sensor schematically shown with the cap die separated from the SOI sensor die.

The relative change of the resistivity $\Delta \rho^{\prime}{ }_{11} / \rho$ along the longitudinal direction of a piezoresistor is given by [2]

$$
\frac{\Delta \rho_{11}^{\prime}}{\rho}=\pi_{11}^{\prime} \sigma_{11}^{\prime}+\pi_{12}^{\prime} \sigma_{22}^{\prime}+\pi_{16}^{\prime} \sigma_{12}^{\prime} \approx \pi_{11}^{\prime} \sigma_{11}^{\prime}+\pi_{12}^{\prime} \sigma_{22}^{\prime},
$$

where $\pi_{11}^{\prime}, \pi_{12}^{\prime}$ and $\pi_{16}^{\prime}$ are the respective longitudinal, transverse and shear piezoresistive coefficients and $\sigma_{11}^{\prime}, \sigma_{22}^{\prime}$ and $\sigma_{12}^{\prime}$ are the respective longitudinal, transverse, and shear stress components. Furthermore, $\sigma_{12}^{\prime}$ is small enough to be neglected under hydrostatic pressure loading conditions [2]. 
For the piezoresistors isolated and bound by the completelyfilled narrow trenches, the relative change in the longitudinal resistivity is given by (1). For those isolated by the partially-filled wide trenches, $\sigma_{22}^{\prime}$ can be largely relieved by a proper design of the trench dimensions, thus reducing the biaxial stress field to a uniaxial stress field. In this case, the relative change in the longitudinal resistivity is given by

$$
\frac{\Delta \rho^{\prime}{ }_{11}}{\rho} \approx \pi_{11}^{\prime} \sigma_{11}^{\prime}
$$

A 2-dimensional finite-element analysis was carried out to investigate the dependence of $\sigma_{22}^{\prime}$ on the dimensions of the trench and the piezoresistor (Fig. 3). It is concluded that the width $w$ of the piezoresistor should be $\leq 3$ times the depth $h$ of the trench in order to effectively relieve the transverse stress $\sigma_{22}^{\prime}$ on the piezoresistor.

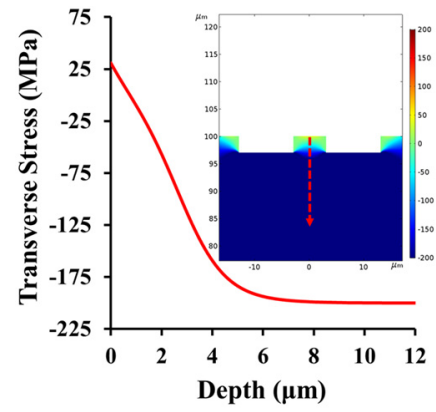

(a)

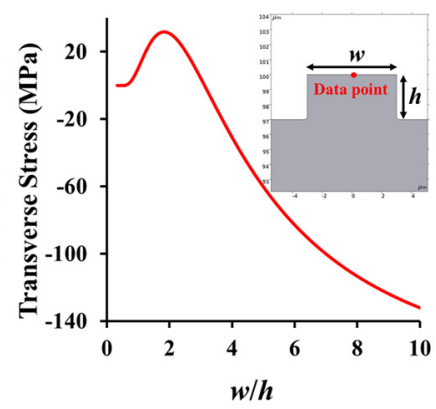

(b)
Figure 3: 2-dimensional finite-element analysis of a trenched piezoresistor under 200-MPa lateral compression. (a) The transverse compressive stress is plotted along the dotted path from the top and (b) the dependence of the transverse stress on $w / h$ at the top of a piezoresistor defined by two wide trenches.

Under an applied hydrostatic pressure $P$ of $200 \mathrm{MPa}$, the simulated values of $\sigma_{[1 \overline{1} 0]}$ and $\sigma_{[001]}$ are respectively -126 and -100 $\mathrm{MPa}$ around the center of the die where the piezoresistors are located [2]. The relative sensitivity of the pressure sensor, estimated and summarized in Table I, is proportional to the magnitude of the difference $\left|\Delta\left(\frac{\Delta \rho}{\rho}\right)\right|$ between the $\frac{\Delta \rho}{\rho}$ of the two orthogonal pairs of piezoresistors: $R_{1}$ and $R_{3}$ aligned to the [001] orientation, and $R_{2}$ and

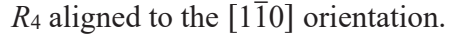

Table I: Relative pressure sensitivity $\left|\Delta\left(\frac{\Delta \rho}{\rho}\right)\right|$ for all combinations of wide and narrow trenches in both p-and n-type piezoresistors.

\begin{tabular}{|c|c|c|c|}
\hline$R_{1}, R_{3}$ & $R_{2}, R_{4}$ & $\begin{array}{c}\left|\Delta\left(\frac{\Delta \rho}{\rho}\right)\right|(\%) \\
(\mathrm{p}-\text { Type })\end{array}$ & $\begin{array}{c}\left|\Delta\left(\frac{\Delta \rho}{\rho}\right)\right|(\%) \\
(\mathrm{n}-\text { Type })\end{array}$ \\
\hline Wide & Wide & 8.4 & 6.3 \\
\hline Narrow & Narrow & 8.4 & 4.9 \\
\hline Narrow & Wide & 8.5 & 0.4 \\
\hline Wide & Narrow & 8.3 & 11.6 \\
\hline
\end{tabular}

It can be seen that wide and narrow trenches have little impact on the pressure sensitivity for an implementation with p-type piezoresistors. The impact is, however, significant for an implementation with n-type piezoresistors for which the highest sensitivity is obtained by having wide trenches on $R_{1}$ and $R_{3}$, and narrow trenches on $R_{2}$ and $R_{4}$. With this configuration the sensitivity of an implementation using n-type piezoresistors can be $\sim 1.4$ times higher than that of an implementation using p-type piezoresistors.

A schematic layout of the sensor and trench designs for the best n-type piezoresistor configuration is shown in Fig. 4. Implemented are $R_{1}$ and $R_{3}$ defined by $10-\mu \mathrm{m}$ wide trenches, and $R_{2}$ and $R_{4}$ by $1-$ $\mu \mathrm{m}$ narrow trenches.

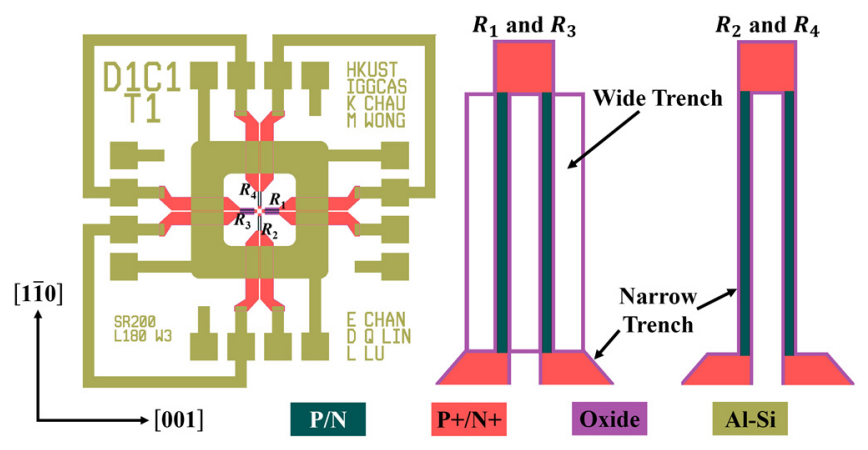

Figure 4: Sensor layout showing piezoresistors $R_{2}$ and $R_{4}$ defined by completely-filled narrow trenches and piezoresistors $R_{1}$ and $R_{3}$ defined by partially-filled wide trenches for the relief of the transverse stress.

\section{FABRICATION}

Sensor fabrication started with an SOI wafer with a $3-\mu \mathrm{m}$ thick [110]-oriented device layer. $1-\mu \mathrm{m}$ and $10-\mu \mathrm{m}$ wide trenches were etched on the device layer reaching the buried oxide layer (Fig. 5a). These were subsequently oxidized to define the piezoresistor active areas. Incidentally, a small notch (Fig. 6) was formed on the top of the oxide-filled narrow trenches resulting from the oxidation process. A blanket boron implantation at an energy of $27 \mathrm{keV}$ and dose of $2.6 \times 10^{15} \mathrm{~cm}^{-2}$, and a second selective boron implantation at the same energy but a higher dose of $2 \times 10^{16} \mathrm{~cm}^{-2}$ were performed, respectively to adjust the doping level of the p-type piezoresistors and to form the P+ electrical leads (Fig. 5b). A 200-nm thick cover silicon dioxide was then deposited by low-pressure chemical vapor deposition, prior to the activation of the implanted dopants at 1000 ${ }^{\circ} \mathrm{C}$ for $30 \mathrm{~min}$. Contact holes were opened through the cover oxide layer and a $1.5-\mu \mathrm{m}$ aluminum-silicon (Al-Si) alloy was deposited and patterned to form the contact pads and the bond ring for the subsequent aluminum-germanium (Al-Ge) eutectic bonding.

A separate silicon cap wafer was prepared, on which $200-\mu \mathrm{m}$ deep cavities were formed using deep reactive-ion etching and subsequently covered with a 500-nm thick evaporated Ge (Fig. 5c). Eutectic bonding of the device and cap wafers (Fig. 5d) was carried out under a force load of $40 \mathrm{kN}$ at $430{ }^{\circ} \mathrm{C}$ for $5 \mathrm{~min}$ in vacuum. The eutectic melt was able to overcome the topography of the small notch, thus resulting in hermetic sealing of the cavity. The $2.5 \times 2.5$ $\mathrm{mm}^{2}$ sensor die (Fig. 7) was packaged after dicing and tested in a custom setup [2].

The same process could be applied to fabricate sensors with ntype piezoresistors by changing the implanted species from boron to phosphorus, energy to $50 \mathrm{keV}$, and implant doses to $10^{15}$ and $10^{16}$ $\mathrm{cm}^{-2}$, respectively, for the piezoresistors and the $\mathrm{N}+$ electrical leads. 


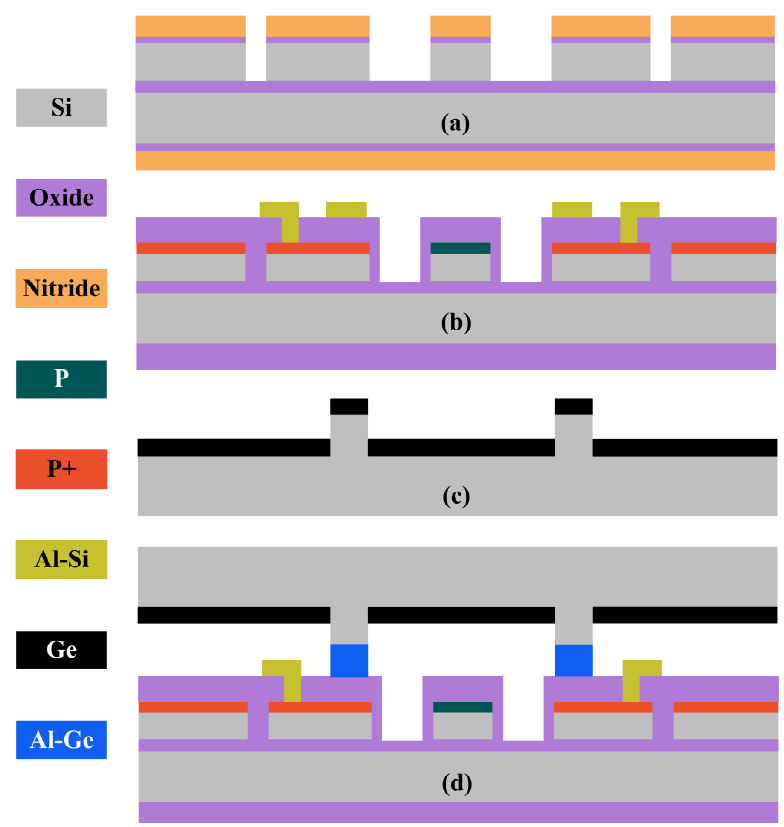

Figure 5: Schematic cross-sections illustrating the fabrication of the SOI pressure sensor having piezoresistors with narrow and wide trenches. (a), (b) SOI wafer, (c) cap wafer, and (d) Al-Ge bonding.

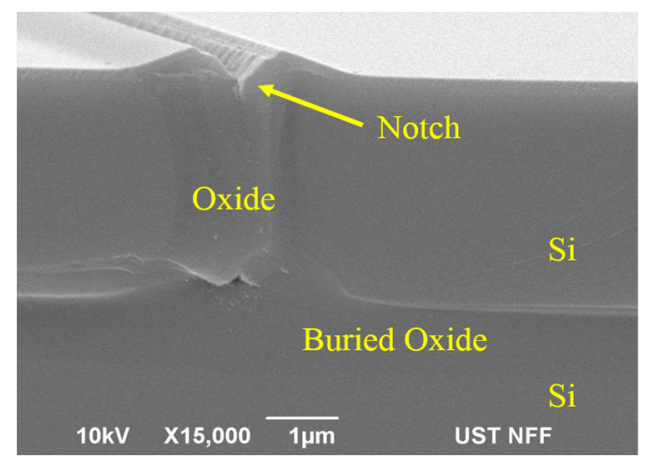

Figure 6: SEM micrograph of the oxide-filled narrow trench.

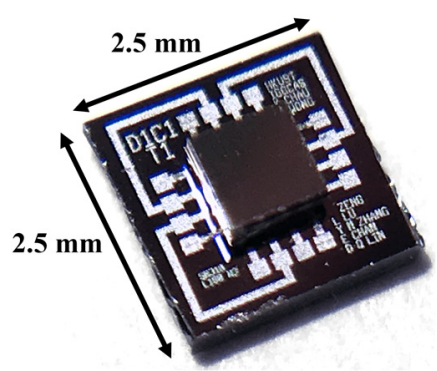

Figure 7: Photomicrograph of the fabricated SOI pressure sensor die.

\section{TEST RESULTS}

SOI pressure sensor with p-type piezoresistors was first realized. The pressure sensor was subjected to an applied hydrostatic pressure $P$ and the dependence of the resistance of individual piezoresistors on $P$ was characterized at room temperature. The test results shown in Fig. 8 are from a pressure sensor in which all four p-type piezoresistors are defined by wide trenches. Consistent with the theory [2], $R_{1}$ and $R_{3}$ aligned with the [001] orientation are almost independent of $P$, whereas $R_{2}$ and $R_{4}$ aligned with the [1 $\left.\overline{1} 0\right]$ orientation are sensitive to $P$. The resistance of $R_{2}$ and $R_{4}$ decreases by $\sim 2.8 \%$ over the $200 \mathrm{MPa}$ range of applied $P$.

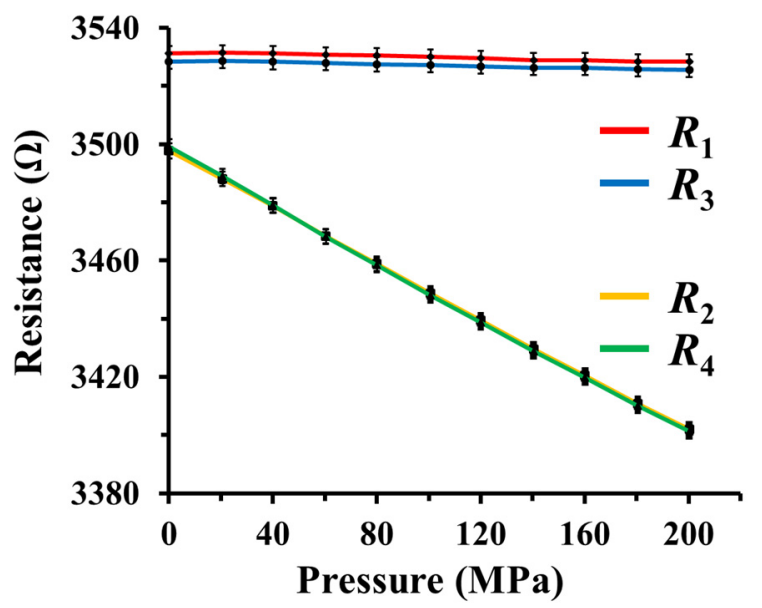

Figure 8: Dependence of the resistance of individual piezoresistors on the applied pressure at room temperature for the SOI pressure sensor with p-type piezoresistors all defined by wide trenches.

The measured Wheatstone bridge output voltage VOUT of the SOI pressure sensor versus $P$ and temperature is shown in Fig. 9. The discontinued plot at $225^{\circ} \mathrm{C}$ was due to a pressure seal failure of the test-package beyond $100 \mathrm{MPa}$ at which point the pressure sensor die was still functional. A room-temperature sensitivity of 66 $\mu \mathrm{V} / \mathrm{V} / \mathrm{MPa}$ over $200 \mathrm{MPa}$ and a temperature coefficient of sensitivity of $-1000 \mathrm{ppm} /{ }^{\circ} \mathrm{C}$ over $200{ }^{\circ} \mathrm{C}$ were obtained. On the other hand, the leakage current of the present dielectric-isolated pressure sensor remained low, reaching only $0.1 \mathrm{nA}$ at $175^{\circ} \mathrm{C}$ (Fig. 10a). This is less than one thousandth of the leakage current of a junction-isolated pressure sensor (Fig. 10b). A comparison of the sensor characteristics of this work and others is shown in Table II.

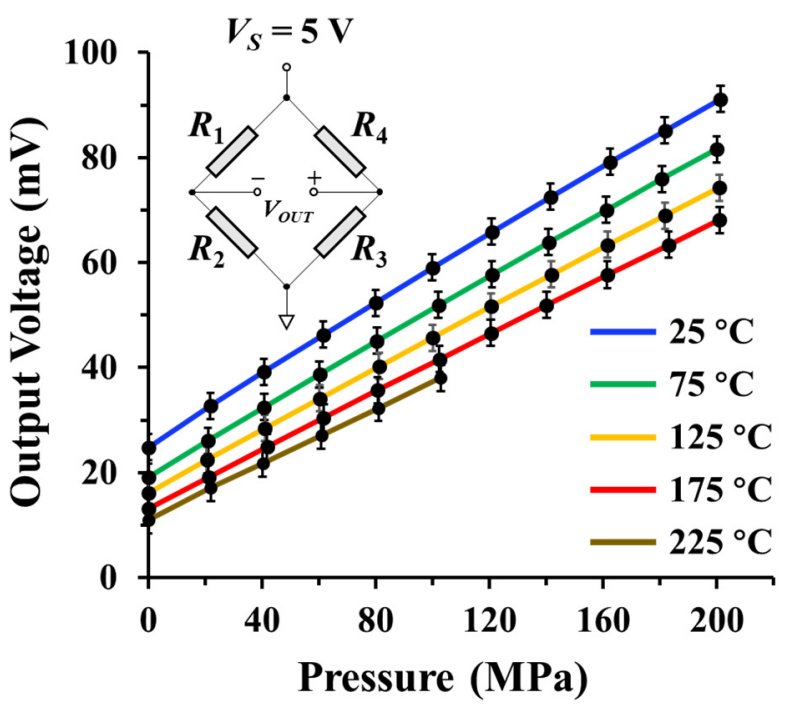

Figure 9: Wheatstone bridge output voltage of the SOI pressure sensor with p-type piezoresistors all defined by wide trenches. 

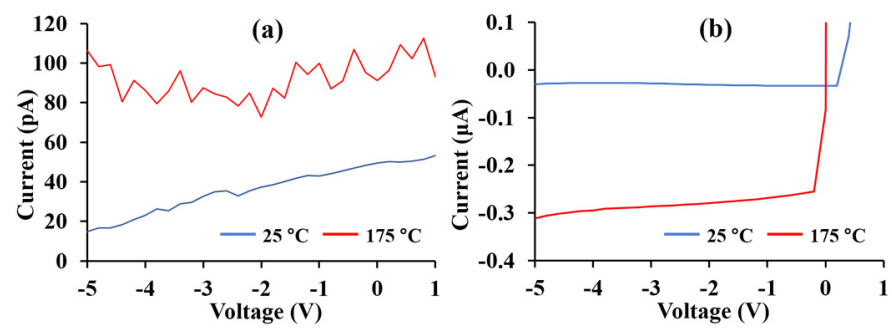

Figure 10: Leakage current of (a) dielectric- and (b) junctionisolated pressure sensors.

Table II: MEMS piezoresistive high-pressure sensor comparison.

\begin{tabular}{|c|c|c|c|c|c|}
\hline Ref. & Type & $\begin{array}{c}\boldsymbol{P}_{\max } \\
(\mathbf{M P a})\end{array}$ & $\begin{array}{c}\Delta \boldsymbol{V}_{\text {out }} \\
(\mathbf{m V})\end{array}$ & $\begin{array}{c}\text { Power } \\
\text { Supply }\end{array}$ & $\begin{array}{c}\text { Temp. } \\
\left({ }^{\circ} \mathbf{C}\right)\end{array}$ \\
\hline$[1]$ & All-Si Bulk & 120 & 58 & $5 \mathrm{~V}$ & $25 \sim 125$ \\
\hline$[2]$ & All-Si Bulk & 200 & 79 & $5 \mathrm{~V}$ & $25 \sim 175$ \\
\hline$[3]$ & $\begin{array}{c}\text { Si-Glass } \\
\text { Diaphragm }\end{array}$ & 150 & 50 & $5 \mathrm{~V}$ & $20 \sim 150$ \\
\hline$[4]$ & $\begin{array}{c}\text { Si-Glass } \\
\text { Diaphragm }\end{array}$ & 150 & 167 & $1.5 \mathrm{~mA}$ & $\leq 200$ \\
\hline$[5]$ & $\begin{array}{c}\text { Si-Glass Bulk } \\
{[6]}\end{array}$ & 200 & 22 & $5 \mathrm{~V}$ & $\mathrm{~N} / \mathrm{A}$ \\
\hline Si-Glass Bulk & 60 & 62 & $5 \mathrm{~V}$ & $-40 \sim 125$ \\
\hline This Work & SOI Bulk & 200 & 66 & $5 \mathrm{~V}$ & $25 \sim 225$ \\
\hline
\end{tabular}

\section{DISCUSSION AND CONCLUSION}

A dielectric-isolated bulk-type pressure sensor on SOI with the piezoresistors defined by isolation trenches was designed and fabricated. Theoretical analysis shows that selective trenching on $\mathrm{p}$ or n-type piezoresistors should result in appreciable difference in the pressure sensitivity, with n-type piezoresistors having better performance. Finite-element analysis shows that the applied stress acting on the piezoresistors could be biaxial or uniaxial, depending on the design of the trenches.
The fabricated sensor with p-type piezoresistors was characterized with pressure loading up to $200 \mathrm{MPa}$ and temperature up to $225^{\circ} \mathrm{C}$. The respective sensitivity and temperature coefficient of sensitivity are $\sim 66 \mu \mathrm{V} / \mathrm{V} / \mathrm{MPa}$ and $-1000 \mathrm{ppm} /{ }^{\circ} \mathrm{C}$. The high temperature performance of the SOI pressure sensor was experimentally demonstrated. The leakage current of the present dielectric-isolated pressure sensor was $0.1 \mathrm{nA}$ at $175^{\circ} \mathrm{C}$, which is less than one thousandth of the leakage current of a junction-isolated pressure sensor.

\section{ACKNOWLEDGMENT}

This work was supported by the Key Research Program of Chinese Academy of Sciences, Grant No. ZDRW-ZS-2016-1-3, and the Strategic Priority Research Program of Chinese Academy of Sciences, Grant No. XDA14040202.

Device fabrication was carried out at the Nanosystem Fabrication Facility (NFF) of the Hong Kong University of Science and Technology.

\section{REFERENCES}

[1] D. Lin, E. Chan, L. Lu, S. Guo, F. Zeng, Y. Zhang, M. Wong, and K. Chau, "A 1200-atmosphere bulk-type all-silicon pressure sensor," in Proc. Transducers, Kaohsiung, Taiwan, Jun. 2017, pp. 2119-2122.

[2] E. Chan, D. Lin, L. Lu, D. Zhang, S. Guo, Y. Zhang, K. Chau, and M. Wong, "Realization and characterization of a bulk-type all-silicon high pressure sensor," J. Microelectromech. Syst., vol. 27, no. 2, pp. 231-238, 2018.

[3] T. Toriyama, K. Sawa, Y. Tanimoto, and S. Sugiyama, "A new piezoresistive high pressure sensor utilizing combination of three-axis normal stress components," in Proc. Int. Symp. Micromechatronics Human Sci., Nagoya, Japan, Nov. 1999, pp. 215-220.

[4] Z. Niu, Y. Zhao, and B. Tian, "Design optimization of high pressure and high temperature piezoresistive pressure sensor for high sensitivity,” Rev. Sci. Instrum., vol. 85, no. 1, p. 015001, 2014.

[5] P. Heinickel and R. Werthschützky, "Functionality of a novel overload resistant silicon high pressure sensing element," in Proc. Transducers, Denver, CO, USA, Jun. 2009, pp. 252-255.

[6] M. Baumann, P. Ruther, and O. Paul, "Modeling and characterization of a CMOS sensor with surface trenches for high-pressure applications," in Proc. MEMS, Cancun, Mexico, Jan. 2011, pp. 601-604. 